\title{
Adaptability of Kinetic Parameters to a Narrow Speed Range in Healthy Free-Moving Cats
}

\author{
Congzhen Liu, ${ }^{1,2}$ Guolin Wang $\mathbb{D}^{1},{ }^{1}$ Haichao Zhou $\mathbb{D}^{1},{ }^{1}$ Ye Mei, ${ }^{1}$ and Bentil M. K. Asafo-Duho ${ }^{1}$ \\ ${ }^{1}$ School of Automotive and Traffic Engineering, Jiangsu University, Zhenjiang 212013, China \\ ${ }^{2}$ School of Transportation and Vehicle Engineering, Shandong University of Technology, Zibo 255049, China \\ Correspondence should be addressed to Guolin Wang; glwang@ujs.edu.cn
}

Received 28 October 2019; Revised 14 April 2020; Accepted 28 April 2020; Published 12 May 2020

Academic Editor: Zhiyun Lin

Copyright ( $\odot 2020$ Congzhen Liu et al. This is an open access article distributed under the Creative Commons Attribution License, which permits unrestricted use, distribution, and reproduction in any medium, provided the original work is properly cited.

Ground reaction force and impulse measurement is an established method for assessing the physical condition and efficacy of treatment for some animals. The primary aim of this study was to quantify the kinetic parameters of the limbs of healthy cats at different speeds. The second aim was to determine the explicit relationship between the kinetic parameters and speed. Peak vertical force (PVF), vertical impulse (VI), stance phase duration (SPD), and paw contact area (PCA) of each limb (forelimb, F; hindlimb, $\mathrm{H}$ ) of seven clinically healthy, client-owned cats were recorded in the speed interval of $0.5-2.5 \mathrm{~m} / \mathrm{s}$. The cats were encouraged to pass by a pressure-sensitive walkway with different speeds. The results revealed that there were no significant differences in any of the tested parameters between the left and right forelimbs and the left and right hindlimbs. Means and regression formulas of the kinetic parameters with speed were obtained. It was evident that PVF-F and PVF-H increased linearly with speed, while VI-F and VI-H decreased exponentially. SPD-F was practically equal to SPD-H and exponentially decreased with speed. PCA-F increased linearly with speed, and PCA-H was almost invariable. Pressure-sensitive walkway is a suitable and convenient equipment for assessing the kinetic parameters of cats. Variation of these kinetic parameters with speed can be explained by the functional difference for forelimb-dominant and hindlimb-driven.

\section{Introduction}

Ground reaction forces and impulses are used to assess lameness induced by diseases (such as osteoarthritis, diabetes, and Parkinson's disease) and the effect of treatment on affected individuals [1-4]. This effective method has also been adopted for some animals to evaluate their health condition and motion characteristics. Force plate analysis has been used frequently to estimate the kinetic parameters of dogs in a noninvasive manner $[5,6]$. With the objective measurement provided by force plate analysis, researchers can acquire the motion characteristics of dogs. In addition, force plate analysis has also been used to evaluate the efficacy of treatments for perioperative or chronic pain associated with osteoarthritis [7-9]. Furthermore, the objective measurement can provide inspiration for engineering bionics such as quadruped robots $[10,11]$. Owing to the reliability and effectiveness of this method, force plate analysis has become an objective standard in the evaluation of canine locomotion.

Cats have been selected as dynamic research objects of several experimental studies that concentrate on the movement of body segment, posture and equilibrium, organization of muscle reflexes during locomotion, and joint mechanics [12-14]. Many studies have focused on feline osteoarthritis for several reasons. Up to $30 \%$ cats suffer from osteoarthritis, and its prevalence increases dramatically with age; however, this disease is neglected in most cases $[15,16]$. In addition, diagnosis of cats is challenging, as cats barely tolerate complete orthopedic examinations $[17,18]$. Furthermore, the severity of lameness and pain cannot be determined reliably using radiographs or orthopedic examination, as these are not in accordance with the results derived from force plate analysis $[19,20]$.

Although the combination of force plate with special equipment has been used in several published studies, this 
system was not conveniently adopted for studying cats [21-23]. Cats are smaller in size than dogs, and their gait length is also shorter. Force platforms cannot readily be used for cats as it must ensure that only one paw contacts the force plate at a time.

The peak vertical force will increase with the increasing speed [24]. However, the explicit relationships of peak vertical force and vertical impulse with speed are still undefined. Additionally, in order to reduce the impact on measurement results, speed was restricted to a narrow interval [25]. Data with speeds more than $2 \mathrm{~m} / \mathrm{s}$ are barely explored.

The first purpose of this study was to quantify the kinetic parameters of healthy cats at different speeds using a pressure-sensitive walkway. The second purpose was to determine the explicit relationships between the kinetic parameters and speed.

\section{Materials and Methods}

2.1. Cat Care and Training. Seven healthy client-owned cats (Chinese Felis silvestris catus) were selected for this study. The group consisted of 3 neutered females, 2 neutered males, and 2 males. The mean age and mean body weight were 3 years and $3.1 \mathrm{~kg}$, respectively. Prior to inclusion in the study, all cats were considered clinically healthy based on physical and orthopedic examinations by veterinarians. The cats were cared for in accordance with the China Animal Health standards. Each one was trained to walk on plywood without stopping, accelerating, and turning around. However, almost all cats expressed fear or curiosity and required time to acclimatize to the procedure. Affection, kibbles, toys, and luminous spots were used to encourage them to attain the desired walking scheme $[24,26]$. Before data collection, all cats were accommodated in the testing room for one week. The feeding, sport, and training activities before testing were performed in this week. This study was approved by the Mechanics Laboratory of Jiangsu University, and owner consent was obtained for each cat enrolled in this study [27].

2.2. Equipment and Data Collection. Kinetic parametric data were collected using a pressure-sensitive walkway (Medical Sensor 5101QL Animal Walkway System, Tekscan), which was $447.0 \mathrm{~mm}$ long, $111.8 \mathrm{~mm}$ wide, and $0.102 \mathrm{~mm}$ thick. The entire pressure-sensitive walkway surface was covered with a thin, nonslip rubber mat to hide the measuring area from the cats' sight, protect the equipment, and prevent slipping [28]. To ensure constant speed of each trial, the walkway was embedded in the middle of a $6.0 \mathrm{~m}$ long flush runway. In addition, two transparent polyvinyl chloride (PVC) fences were set on both sides of the runway to guide the animals. Figure 1 shows the measurement system. Equilibration and calibration were performed prior to data acquisition according to the specifications. The sampling rate for this system was $100 \mathrm{~Hz}$ [25]. Prior to data collection, the body weight of each cat was determined on an electronic scale.
The speed was not restricted in the data collection process, so the cats could move ahead freely with different speeds (free-moving cats). To determine the speed and acceleration of each trial, a high-speed video camera (Olympus i-Speed) was used in synchrony with the pressuresensitive walkway. The optical axis of the camera was set perpendicular to the straight line of the cats walking along the walkway. Figure 2 shows a frame of the stored data from the high-speed video camera. The sampling rate was $100 \mathrm{fps}$ [22]. A single observer evaluated each foot strike and determined the validity of the trial [29].

2.3. Data Analysis. Peak vertical force (PVF), vertical impulse (VI), stance phase duration (SPD), and paw contact area (PCA) were processed using the Tekscan proprietary software (Walkway 7.70). PVF and VI were normalized to individual cat's body weight (BW) and expressed as \% BW and $\% \mathrm{BW} \cdot \mathrm{s}$, respectively $[30,31]$. The range of the measured speed was $0.4-2.7 \mathrm{~m} / \mathrm{s}$, and the adopted data were in the interval of $0.5-2.5 \mathrm{~m} / \mathrm{s}$. PVF, VI, SPD, and PCA were calculated and presented as means \pm standard deviation.

2.4. Statistical Analysis. Scatter diagrams were initially used to estimate data variation tendency. Three types of statistical analyses were performed to assess differences among each pair of paws and potential correlativity of the kinetic parameters. The Mann-Whitney test was used to compare the left and right forelimbs, as well as the left and right hindlimbs. Means, standard deviations, Mann-Whitney $U$ statistics, and the corresponding $P$ values were calculated. This method was also adopted for comparing the forelimbs and hindlimbs. Correlation analysis (Pearson's correlation coefficient $r$ ) was implemented to evaluate the potential correlation between PVF, VI, SPD, PCA, and speed. Regression analysis was performed to test the probable relationships of PVF, VI, SPD, and PCA with speed. Statistical significance was set at $P<0.05$ for all tests.

\section{Results}

Means of all trials at speeds between $0.50 \mathrm{~m} / \mathrm{s}$ and $2.5 \mathrm{~m} / \mathrm{s}$, symmetry ratio, and results of the Mann-Whitney test are shown in Table 1. The right and left forelimbs constituted a pair, while the right and left hindlimbs constituted another pair. PCA denoted the maximum contact area during the contact phase. There were no significant differences between the kinetic parameters of the left and right forelimbs and the left and right hindlimbs, as each symmetry ratio was almost equal to 1, and the significance levels (Sig.1) of all pairs were more than 0.05 . The left and right pooled data were used in subsequent statistical analyses. Results of the further Mann-Whitney test indicated that the PVF and VI of forelimbs were significantly higher than those of the hindlimbs, for each $P$ value was less than 0.001 . As for SPD, there was no significant difference between forelimbs and hindlimbs $(P=0.57)$. The PCA of forelimbs was statistically higher than that of the hindlimbs, but not quite significant $(P=0.01)$. 

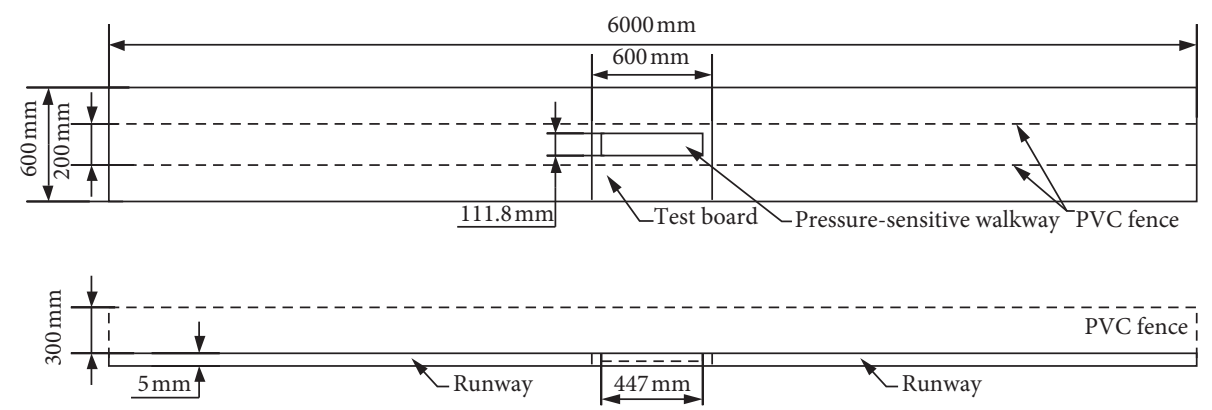

Figure 1: The measuring system for cats.

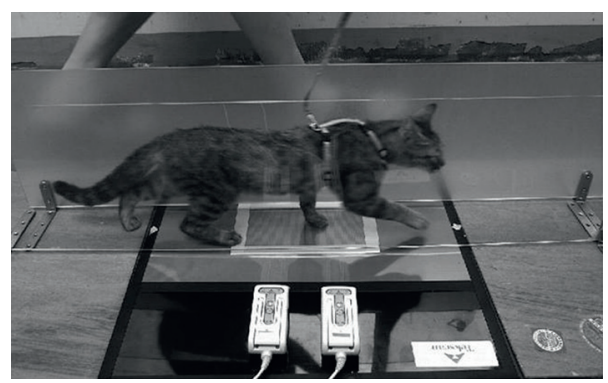

FIGURE 2: Cat traversing the pressure-sensitive walkway.

TABle 1: Means of all trials and results of the Mann-Whitney test.

\begin{tabular}{|c|c|c|c|c|c|c|c|}
\hline \multirow{2}{*}{ Items } & \multirow{2}{*}{ Limb } & \multirow{2}{*}{ Mean \pm SD } & \multirow{2}{*}{ Symmetry ratio } & \multicolumn{2}{|c|}{ Mann-Whitney test } & \multicolumn{2}{|c|}{ Further Mann-Whitney test } \\
\hline & & & & Mann-Whitney $U_{1}$ & Sig.1 & Mann-Whitney $U_{2}$ & Sig. 2 \\
\hline \multirow{3}{*}{ PVF (\% BW) } & RF & $77.31 \pm 16.21$ & $1.03 \pm 0.04$ & 296.00 & 0.75 & \multirow{3}{*}{569.00} & \multirow{3}{*}{$<0.001$} \\
\hline & LF & $76.38 \pm 16.28$ & \multirow[b]{2}{*}{$1.02 \pm 0.03$} & \multirow[b]{2}{*}{298.50} & \multirow[b]{2}{*}{0.79} & & \\
\hline & $\begin{array}{l}\text { RH } \\
\text { LH }\end{array}$ & $\begin{array}{l}61.21 \pm 8.89 \\
61.99 \pm 9.74\end{array}$ & & & & & \\
\hline \multirow{4}{*}{$\mathrm{VI}(\% \mathrm{BW} \cdot \mathrm{s})$} & $\mathrm{RF}$ & $11.09 \pm 3.23$ & \multirow{2}{*}{$0.99 \pm 0.02$} & \multirow{2}{*}{307.00} & \multirow{2}{*}{0.92} & \multirow{4}{*}{614.50} & \multirow{4}{*}{$<0.001$} \\
\hline & $\mathrm{LF}$ & $11.13 \pm 3.33$ & & & & & \\
\hline & RH & $8.96 \pm 3.22$ & \multirow{2}{*}{$1.02 \pm 0.03$} & \multirow{2}{*}{296.50} & \multirow{2}{*}{0.61} & & \\
\hline & $\mathrm{LH}$ & $9.24 \pm 3.28$ & & & & & \\
\hline \multirow{4}{*}{ SPD (s) } & $\mathrm{RF}$ & $0.26 \pm 0.11$ & & 31000 & 096 & \multirow{4}{*}{1168.00} & \multirow{4}{*}{0.57} \\
\hline & LF & $0.26 \pm 0.11$ & $1.01 \pm 0.02$ & & 0.90 & & \\
\hline & RH & $0.26 \pm 0.11$ & \multirow[b]{2}{*}{$1.01 \pm 0.01$} & \multirow{2}{*}{307.50} & \multirow{2}{*}{0.92} & & \\
\hline & $\mathrm{LH}$ & $0.27 \pm 0.13$ & & & & & \\
\hline \multirow{4}{*}{ PCA $\left(\mathrm{mm}^{2}\right)$} & $\mathrm{RF}$ & $542.76 \pm 43.60$ & \multirow{2}{*}{$1.05 \pm 0.05$} & \multirow{2}{*}{302.00} & \multirow{2}{*}{0.84} & \multirow{4}{*}{873.50} & \multirow{4}{*}{0.01} \\
\hline & $\mathrm{LF}$ & $539.12 \pm 41.17$ & & & & & \\
\hline & RH & $524.80 \pm 28.65$ & \multirow{2}{*}{$0.98 \pm 0.03$} & \multirow{2}{*}{255.00} & \multirow{2}{*}{0.26} & & \\
\hline & LH & $513.92 \pm 33.76$ & & & & & \\
\hline
\end{tabular}

RF, right forelimb; LF, left forelimb; RH, right hindlimb; LH, left hindlimb; SD, standard deviation; symmetry ratio, left/right; Sig.1 and Sig.2, significance level.

The results of correlation analysis and regression analysis about pooled data are shown in Table 2. All the absolute values of Pearson's correlation coefficients were more than 0.84 , with the exception of PCA-H. In addition, all $P$ values were lesser than 0.001 , other than that for PCA-H. These results suggested that speed correlated strongly with other kinematic parameters with the exception of PCA-H. High correlation coefficients manifested the rationality of regression analysis except PCA-H. Furthermore, speed showed positive relationship with PVF-F, PVF-H, and PCA-F and negative relationship with VI-F, VI-H, SPD-F, and SPD-H.
Regression analysis was performed to further probe the relationships between speed and all other kinematic parameters with the exception of PCA-H. Scatter diagrams and regression curves are shown in Figures 3-6. Regression formulas and coefficients of determination $\left(R^{2}\right)$ are shown in Table 2.

As the speed increased from $0.5 \mathrm{~m} / \mathrm{s}$ to $2.5 \mathrm{~m} / \mathrm{s}$, the span of PVF-F and PVF-H was $52-103 \%$ BW and $46-71 \%$ BW, respectively. Scatter diagrams of PVF-F and PVF-H with speed showed an almost linear relationship because of which linear regression was used (Figure 3). In addition, the coefficients of determination were 0.95 and 0.81 for these two 
TABLE 2: Results of correlation analysis and regression analysis about pooled data.

\begin{tabular}{|c|c|c|c|c|c|}
\hline \multirow{2}{*}{ Items } & \multirow{2}{*}{ Limb } & \multicolumn{2}{|c|}{ Correlation analysis } & \multicolumn{2}{|c|}{ Regression analysis } \\
\hline & & $r$ & Sig. (2-tailed) & Formula & $R^{2}$ \\
\hline \multirow{2}{*}{ PVF (\% BW) } & $\mathrm{F}$ & 0.98 & $<0.001$ & $32.21 V+29.56$ & 0.95 \\
\hline & $\mathrm{H}$ & 0.84 & $<0.001$ & $19.96 V+29.66$ & 0.81 \\
\hline \multirow{2}{*}{ VI (\% BW·s) } & $\mathrm{F}$ & -0.90 & $<0.001$ & $13.41 V^{-0.70}$ & 0.96 \\
\hline & $\mathrm{H}$ & -0.86 & $<0.001$ & $11.19 \mathrm{~V}^{-0.79}$ & 0.91 \\
\hline \multirow{2}{*}{ SPD (s) } & $\mathrm{F}$ & -0.92 & $<0.001$ & $0.33 V^{-1.00}$ & 0.98 \\
\hline & $\mathrm{H}$ & -0.89 & $<0.001$ & $0.34 V^{-1.01}$ & 0.95 \\
\hline \multirow{2}{*}{ PCA $\left(\mathrm{mm}^{2}\right)$} & $\mathrm{F}$ & 0.92 & $<0.001$ & \multirow{2}{*}{$73.18 V+432.92$} & \multirow{2}{*}{0.85} \\
\hline & $\mathrm{H}$ & 0.14 & 0.52 & & \\
\hline
\end{tabular}

F, forelimb; H, hindlimb; $r$, Pearson's correlation coefficients; Sig. (2-tailed), two-tailed significance level; $V$, speed (m/s); $R^{2}$, coefficient of determination.

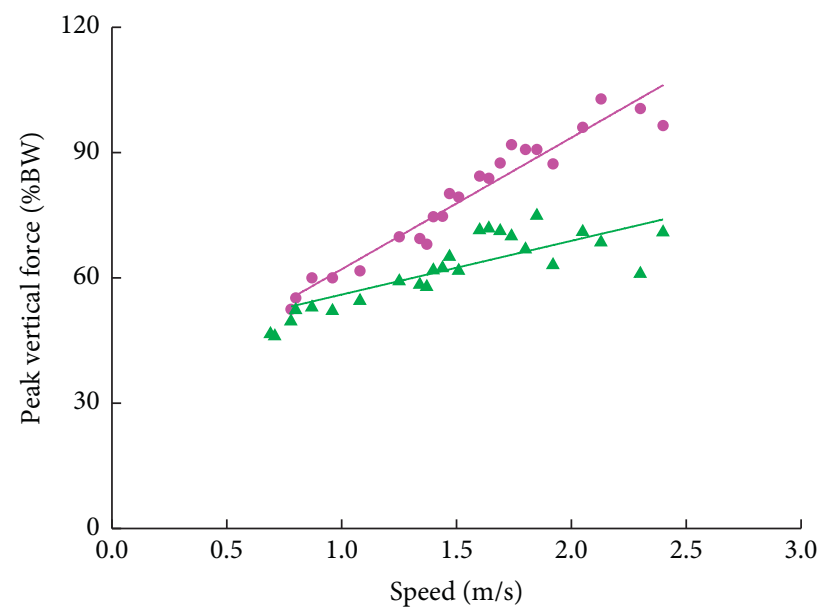

$\Delta$ Forelimb

- Hindlimb

FiguRE 3: Scatter diagrams and regression curves of PVF versus speed.

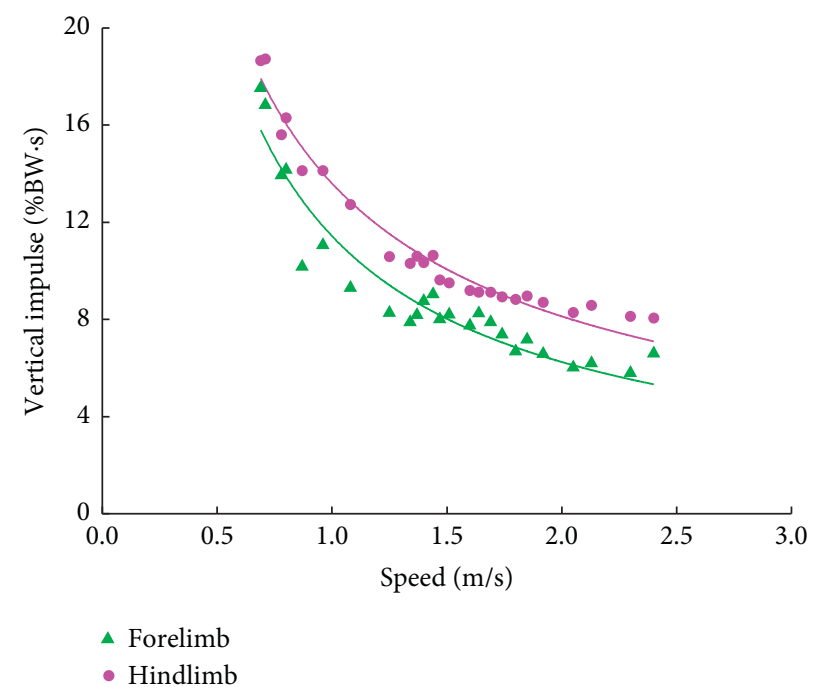

FIgURE 4: Scatter diagrams and regression curves of VI versus speed.

formulas, which indicated credible matching of linear regression. PVF-F was 6-30\% BW higher than PVF-H within the speed range. The same method was used for PCA-F, with 0.85 coefficient of determination.
VI-F and VI-H decreased from $0.19 \% \mathrm{BW} \cdot \mathrm{s}$ to $0.08 \%$ $\mathrm{BW} \cdot \mathrm{s}$ and from $0.18 \% \mathrm{BW} \cdot \mathrm{s}$ to $0.06 \% \mathrm{BW} \cdot \mathrm{s}$, respectively, within the speed range. The exponential fitting method was used on the basis of the tendency observed in the scatter 


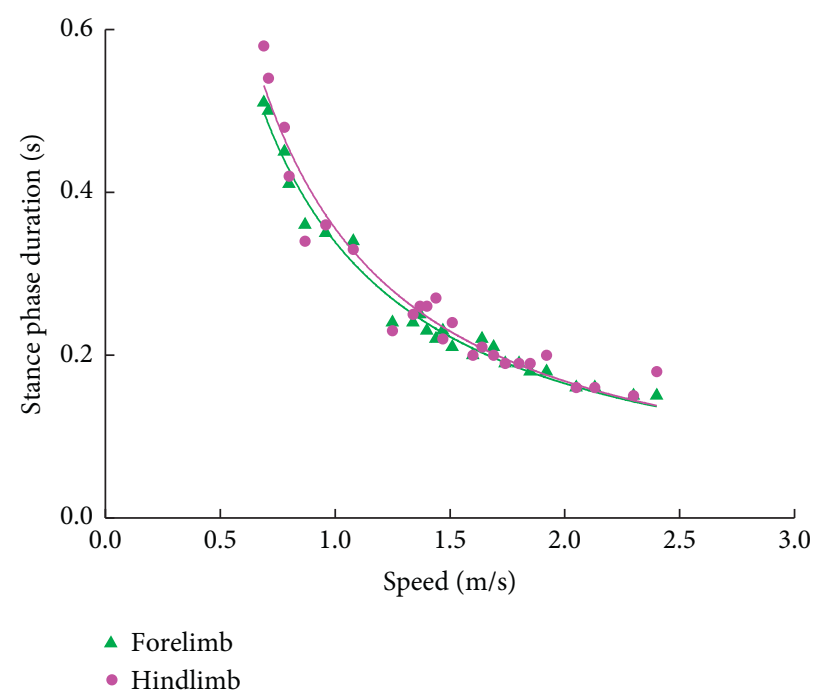

FIGURE 5: Scatter diagrams and regression curves of SPD versus speed.

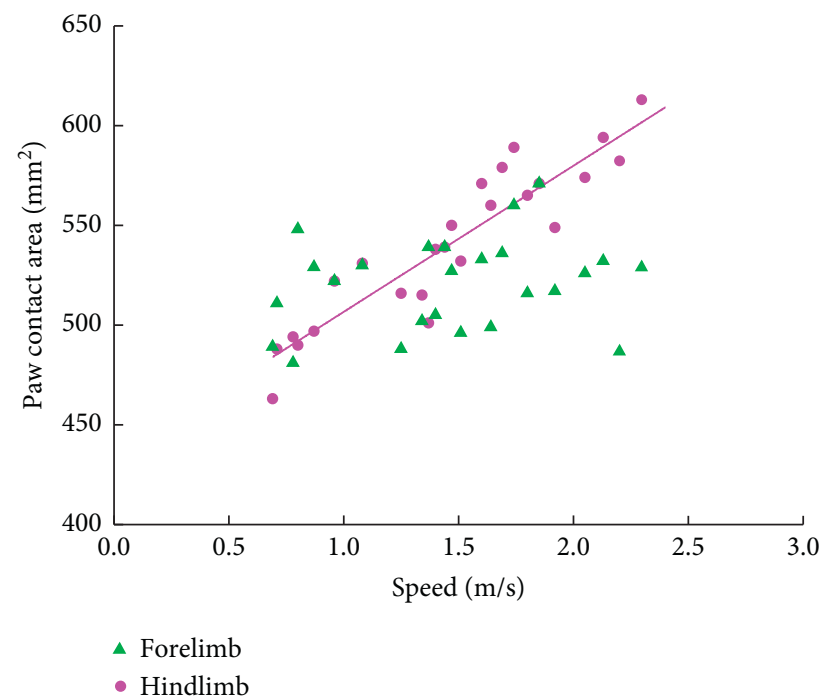

Figure 6: Scatter diagrams and regression curves of PCA versus speed.

diagrams. This method was desirable as both the coefficients of determination were more than 0.91. As the tendency of both SPD-F and SPD-H was almost similar to that of VI according to the scatter diagrams, the exponential regression method was selected once more for SPD. The high coefficients of determination (0.95 and 0.98) were indicative of perfect regression. Furthermore, the maximum difference between the SPD-F and SPD-H formulas was $4 \%$ in the speed range, which indicated that the SPD-F was almost identical to SPD-H. Thus, a merged and simplified formula was expressed as follows:

$$
\mathrm{SPD}=0.34 V^{-1}
$$

where $V$ is the speed $(\mathrm{m} / \mathrm{s})$.

Figure 6 shows the scatter diagram of PCA-H, and no explicit tendency was discerned from the dispersive distribution.

\section{Discussion}

In this study, the kinetic parameters of seven cats moving at different speeds were collected. They were encouraged to walk across a pressure-sensitive walkway with different speeds. Data were accepted only if the cats traversed the entire walkway in a straight line without any disturbance. To ensure data reliability, readings for paw touching the boundary of the pressure-sensitive walkway were discarded throughout the experiment. Results of statistical analysis revealed the internal relationships of PVF, VI, SPD, and PCA with speed.

The kinetic parameters of the right and left sides were symmetrical, with only minor variations in our results, which is comparable to those reported previously [24, 32]. Symmetry indicated the cats were healthy, as only nonlame cats were included in this study [33]. Moreover, symmetry was the precondition of pooled data for further analysis. The 
PVF and VI of forelimbs were higher than those of the hindlimbs, which is also in agreement with the results of previous studies. A reasonable interpretation for the differences in PVF and VI between forelimbs and hindlimbs is the functional difference, for cats are forelimb-dominant and hindlimb-driven $[28,34]$. Only means of PVF and VI within a speed range from $0.6 \mathrm{~m} / \mathrm{s}$ to $1.1 \mathrm{~m} / \mathrm{s}$ were calculated in previous publications on healthy cats, in which the mean values for PVF-F and PVF-H were $48.0-60.0 \%$ BW and $38.0-50.0 \%$ BW, respectively, while those for VI-F and VI-H were $16.9-19.0 \% \mathrm{BW} \cdot \mathrm{s}$ and $13.0-14.6 \% \mathrm{BW} \cdot \mathrm{s}$ in [35]. To the best of our knowledge, this is the first study to show explicit relationships of PVF and VI with speed. Table 3 shows the comparison of PVF and VI intervals from previous reports and the regression formulas. The results of our study are slightly broader but close to the results of previous reports, which confirms the validity and reliability of the regression formulas. In general, PVF-F and PVF-H increased linearly with speed, while VI-F and VI-H decreased exponentially.

Considering the similarity between the regression formulas of SPD-F and SPD-H with respect to speed, the formulation of a merged and simplified equation is understandable. SPD-F is practically equal to SPD-H and decreases with increasing speed, which is in accordance with the results of other studies [36, 37]. SPD of forelimbs and hindlimbs almost decreased exponentially with speed according to our study. Analogues of exact relationships are scarcely available in previous publications. The values of SPD in previous reports calculated using the regression formula are shown in Table 4. Our results overlap considerably with the previous values with slight variations, which confirms the reliability of the regression formulas.

According to the regression formulas mentioned above, the parameters changed in certain relationships with speed. These parameters are considered to be constant in a narrow speed interval, which is acceptable. Previous studies have obtained the means of these parameters but do not express their relationships with speed, possibly because of a narrow speed interval. In our study, the speed was not restricted, so data of free-moving cats were obtained in a wider speed interval. To the best of our knowledge, this is the first time that data have been obtained at speeds more than $2.0 \mathrm{~m} / \mathrm{s}$ and the widest speed interval $(0.5-2.5 \mathrm{~m} / \mathrm{s})$ in cat research. A significant advantage of the regression formulas is their suitability in a wide speed range.

With the same friction coefficient, the grip force increased with the contact area for hyperelastic materials [38]. An almost linear rise for PCA-F with speed indicated that the forepaws can provide higher braking force and steering force for high-speed motion in cats. This property is beneficial for providing stability and security. As is evident in cats, forelimbs are more important than hindlimbs for weight bearing, maneuvering, and deceleration [39]. In comparison, no explicit relationship of PCA-H with speed can be deduced, as Pearson's correlation coefficient is only 0.14 . To a large extent, PCA-H is invariable in a wide speed interval according to our study.

Different techniques have been used for basic kinetic parametric research, disease, and drug therapy in several species. Force plate analysis is more extensive than other techniques as it generates more comprehensive data [40, 41]. Several studies have used the force plate for kinetic measurements in cats. In each report, a peculiar platform was constructed with suitable length to prevent more than one paw from contacting the platform simultaneously $[19,22,27,42]$. Considering the hardware construction, the short stride length of cats, and the way that the software collects and processes data, a pressure-sensitive walkway is more appropriate than a force plate for cats [43]. One disadvantage of the pressure-sensitive walkway is its inability to capture all the ground reaction forces such as the craniocaudal and mediolateral forces [44]. To the best of our knowledge, this study adopts the densest pressure-sensitive walkway (15.5 sensors per square centimeter) for kinetic parameter measurement of cat pads, which provides more reliable and detailed data.

Speed has a strong impact on other kinematic parameters [45]. Most studies focus on the diagnosis of diseases and analysis of the therapeutic effect of drugs because of which comfortable speeds were selected [46, 47]. Some researchers collected data in a short period after the cats were acclimatized to the environment and equipment. They observed that even without an extensive acclimatization or training period, reliable results can be produced in a uniform population of domestic cats [28]. However, in our experience, reliable data are difficult to obtain after a short period of familiarization because cats move at asymmetrical speeds due to fear, hesitation, crouching, and rolling. Data acquired under this condition do not represent normal behavior. At least two possible aspects are responsible for the objectivity and wide range of the data in our study. First, the cats were sufficiently acclimatized to the environment, manipulators, experimental facilities, and the process before the commencement of research. They could sport in the lab comfortably on their own because of which the data reflected their normal motion. Second, several means such as kibbles, toys, canned food, and affection were used to allure the cats. They passed by the pressure-sensitive walkway at different speeds. The results were discarded for speed variations of more than $0.1 \mathrm{~m} / \mathrm{s}$ in a measurement [39].

The kinetic parameters measured in our study are comparable to those in previous reports, which suggest that the pressure-sensitive walkway can be used to evaluate cats. Measurement results can be used to evaluate clinical cases that suffer from osteoarthritis and evaluate the efficacy of treatments. In future, this approach will be used to estimate the pressure distribution of cat pads.

Discrepancies between our results and those of others may be because of different kinematic patterns between breeds $[29,48]$. Despite our speculations, we must emphasize that the discrepancies may also result from other factors such as speed, acceleration, equipment type, calibration, body weight, and size.

This study still has certain limitations. Owing to the restrictions of the pressure-sensitive walkway, only vertical forces and impulses were analyzed. In fact, craniocaudal and mediolateral forces and impulses, which provide acceleration, braking, and steering forces, are significant factors 
TABLE 3: Comparisons of PVF and VI of the present study and those of previous reports in the same mean speed interval (0.6 1.1 m/s).

\begin{tabular}{lcccc}
\hline & PVF-F (\% BW) & PVF-H $(\%$ BW) & VI-F (\% BW·s) & VI-H (\% BW·s) \\
\hline Previous reports & $48.0 \sim 60.0$ & $38.0 \sim 50.0$ & $19.0 \sim 16.9$ & $14.6 \sim 13.0$ \\
Present study & $48.9 \sim 65.0$ & $41.6 \sim 51.6$ & $19.1 \sim 14.3$ & $16.7 \sim 10.4$ \\
\hline
\end{tabular}

TABLE 4: Comparisons of SPD from previous reports and that calculated in the present study in the same speed interval (0.61 0.67 $\mathrm{m} / \mathrm{s})$.

\begin{tabular}{|c|c|c|c|c|c|c|}
\hline & Speed $(\mathrm{m} / \mathrm{s})$ & Gender & RF (s) & LF (s) & RH (s) & LH (s) \\
\hline \multirow{2}{*}{ Verdugo et al. [24] } & $0.63 \pm 0.06$ & Male & $0.48 \pm 0.08$ & $0.48 \pm 0.07$ & $0.46 \pm 0.07$ & $0.46 \pm 0.09$ \\
\hline & $0.61 \pm 0.04$ & Female & $0.46 \pm 0.06$ & $0.45 \pm 0.07$ & $0.45 \pm 0.06$ & $0.44 \pm 0.08$ \\
\hline Quang et al. [37] & $0.67 \pm 0.22$ & & $0.50 \pm 0.11$ & $0.50 \pm 0.11$ & $048 \pm 0.10$ & $0.49 \pm 0.12$ \\
\hline Present research & $0.61 \sim 0.67$ & & \multicolumn{4}{|c|}{$0.54 \sim 0.49$} \\
\hline
\end{tabular}

determining dynamics and stability [27, 34]. The cat breed was another limitation. The seven cats selected for this study belong to the same breed. Hence, caution should be exercised when applying data from this study because of possible discrepancies between breeds.

\section{Conclusions}

The pressure-sensitive walkway provides an objective estimation of biomechanical characteristics of cats moving at different speeds. There are no significant differences between the left and right forelimbs and the left and right hindlimbs for any of the reported parameters. Based on the results in the speed interval of $0.5-2.5 \mathrm{~m} / \mathrm{s}$, explicit relationships between the kinetic parameters and speed were preliminarily acquired. PVF-F and PVF-H were concluded to increase linearly, while VI-F and VI-H decreased exponentially with speed. SPD-F was practically equal to SPD-H and exponentially decreased with speed. PCA-F increased linearly with speed, compared to the almost invariable PCA-H. The possible interpretation for these different relationships is functional distinction. Cats are forelimb-dominant and hindlimb-driven.

\section{Data Availability}

The data used to support the findings of this study are available from the corresponding author upon request.

\section{Conflicts of Interest}

None of the authors has any other financial or personal relationships that could inappropriately influence or bias the content of the paper.

\section{Acknowledgments}

The authors would like to thank all the owners who allowed their cats to be used for this research. Additionally, the authors thank Dr. Yang Wang, Jichao Sun, Yalong Li, and Yang Yuan for taking care of the animals and their resourcefulness in the data collection process. This study was supported by the National Natural Science Foundation of China (grant nos. 51675240 and 51605198), Jiangsu Youth Fund (grant no. KB20160528), Jiangsu Province Six Talents Summit Program (grant number JXQC-011), and Jiangsu
University Senior Talents Startup Fund Project Funding (grant no. 1291120046).

\section{References}

[1] C. A. Miller and M. C. Verstraete, "Determination of the step duration of gait initiation using a mechanical energy analysis," Journal of Biomechanics, vol. 29, no. 9, pp. 1195-1199, 1996.

[2] R. K. Begg, W. A. Sparrow, and N. D. Lythgo, "Time-domain analysis of foot-ground reaction forces in negotiating obstacles," Gait \& Posture, vol. 7, no. 2, pp. 99-109, 1998.

[3] P. M. Mills, R. S. Barrett, and S. Morrison, "Agreement between footswitch and ground reaction force techniques for identifying gait events: inter-session repeatability and the effect of walking speed," Gait \& Posture, vol. 26, no. 2, pp. 323-326, 2007.

[4] R. M. Kiss, "Comparison between kinematic and ground reaction force techniques for determining gait events during treadmill walking at different walking speeds," Medical Engineering \& Physics, vol. 32, no. 6, pp. 662-667, 2010.

[5] S. J. Hobbs and H. M. Clayton, "Sagittal plane ground reaction forces, centre of pressure and centre of mass in trotting horses," The Veterinary Journal, vol. 198, no. 1, pp. e14-e19, 2013.

[6] N. Volstad, B. Nemke, and P. Muir, "Variance associated with the use of relative velocity for force platform gait analysis in a heterogeneous population of clinically normal dogs," The Veterinary Journal, vol. 207, no. 174, pp. 80-84, 2016.

[7] R. M. Mclaughlin, C. W. Miller, C. L. Taves, T. C. Hearn, N. C. Palmer, and G. I. Anderson, "Force plate analysis of triple pelvic osteotomy for the treatment of canine hip dysplasia," Veterinary Surgery, vol. 20, no. 5, pp. 291-297, 1991.

[8] B. D. X. Lascelles, S. C. Roe, E. Smith et al., "Evaluation of a pressure walkway system for measurement of vertical limb forces in clinically normal dogs," American Journal of Veterinary Research, vol. 67, no. 2, pp. 277-282, 2006.

[9] W. J. Gordon, M. G. Conzemius, E. Riedesel et al., "The relationship between limb function and radiographic osteoarthrosis in dogs with stifle osteoarthrosis," Veterinary Surgery, vol. 32, no. 5, pp. 451-454, 2003.

[10] D. V. Lee, J. E. A. Bertram, and R. J. Todhunter, "Acceleration and balance in trotting dogs," Journal of Experimental Biology, vol. 202, no. 24, pp. 3565-3573, 2000.

[11] L. R. Palmer and D. E. Orin, "3D control of a high-speed quadruped trot," Industrial Robot: An International Journal, vol. 33, no. 4, pp. 298-302, 2006.

[12] F. Caliebe, P. Haussler, P. Hoffmann, M. Illert, J. Schirrmacher, and E. Wiedemann, "Cat distal forelimb 
joints and locomotion: an x-ray study," European Journal of Neuroscience, vol. 3, no. 1, pp. 18-31, 1991.

[13] E. M. Hasler, W. Herzog, T. R. Leonard, A. Stano, and H. Nguyen, "In vivo knee joint loading and kinematics before and after ACL transection in an animal model," Journal of Biomechanics, vol. 31, no. 3, pp. 253-262, 1997.

[14] B. J. Farrell, M. A. Bulgakova, M. G. Sirota, B. I. Prilutsky, and I. N. Beloozerova, "Accurate stepping on a narrow path: mechanics, EMG, and motor cortex activity in the cat," Journal of Neurophysiology, vol. 114, no. 5, pp. 2682-2702, 2015.

[15] S. P. Clarke, D. Mellor, D. N. Clements et al., "Prevalence of radiographic signs of degenerative joint disease in a hospital population of cats," Veterinary Record, vol. 157, no. 25, pp. 793-799, 2005.

[16] B. D. X. Lascelles, J. B. Henry III, J. Brown et al., "Crosssectional study of the prevalence of radiographic degenerative joint disease in domesticated cats," Veterinary Surgery, vol. 39, no. 5, pp. 535-544, 2010.

[17] H. Zamprogno, B. D. Hansen, H. D. Bondell et al., "Item generation and design testing of a questionnaire to assess degenerative joint disease-associated pain in cats," American Journal of Veterinary Research, vol. 71, no. 12, pp. 1417-1424, 2010.

[18] H. C. Kranenburg, B. P. Meij, E. M. L. V. Hofwegen et al., "Prevalence of spondylosis deformans in the feline spine and correlation with owner-perceived behavioural changes," Veterinary and Comparative Orthopaedics and Traumatology, vol. 25, no. 3, pp. 217-223, 2012.

[19] E. Suter, W. Herzog, T. R. Leonard, and H. Nguyen, "Oneyear changes in hind limb kinematics, ground reaction forces and knee stability in an experimental model of osteoarthritis," Journal of Biomechanics, vol. 31, no. 6, pp. 511-517, 1998.

[20] B. D. X. Lascelles, Y.-H. Dong, D. J. Marcellin-Little, A. Thomson, S. Wheeler, and M. Correa, "Relationship of orthopedic examination, goniometric measurements, and radiographic signs of degenerative joint disease in cats," $B M C$ Veterinary Research, vol. 8, no. 1, pp. 10-18, 2012.

[21] J. M. Macpherson, D. W. Lywood, and A. Van Eyken, "A system for the analysis of posture and stance in quadrupeds," Journal of Neuroscience Methods, vol. 20, no. 1, pp. 73-82, 1987.

[22] B. J. Mcfadyen, S. Lavoie, and T. Drew, "Kinetic and energetic patterns for hindlimb obstacle avoidance during cat locomotion," Experimental Brain Research, vol. 125, no. 4, pp. 502-510, 1999.

[23] C. W. Romans, M. G. Conzemius, C. L. Horstman, W. J. Gordon, and R. B. Evans, "Use of pressure platform gait analysis in cats with and without bilateral onychectomy," American Journal of Veterinary Research, vol. 65, no. 9, pp. 1276-1278, 2004.

[24] M. R. Verdugo, S. C. Rahal, F. S. Agostinho, V. M Govoni, M. J. Mamprim, and F. O. Monteiro, "Kinetic and temporospatial parameters in male and female cats walking over a pressure sensing walkway," BMC Veterinary Research, vol. 9, no. 1, pp. 1-7, 2013.

[25] E. Schnabl-Feichter, A. Tichy, and B. Bockstahler, "Coefficients of variation of ground reaction force measurement in cats," PLoS One, vol. 12, no. 3, Article ID e0171946, 2017.

[26] Z. Zhang, J. Yang, and H. Yu, "Effect of flexible back on energy absorption during landing in cats: a biomechanical investigation," Journal of Bionic Engineering, vol. 11, no. 4, pp. 506-516, 2014.
[27] M. Moreau, M. Guillot, J.-P. Pelletier, J. Martel-Pelletier, and É. Troncy, "Kinetic peak vertical force measurement in cats afflicted by coxarthritis: data management and acquisition protocols," Research in Veterinary Science, vol. 95, no. 1, pp. 219-224, 2013.

[28] R. J. Gregor, D. W. Smith, and B. I. Prilutsky, "Mechanics of slope walking in the cat: quantification of muscle load, length change, and ankle extensor EMG patterns," Journal of Neurophysiology, vol. 95, no. 3, pp. 1397-1409, 2006.

[29] M. F. Besancon, M. G. Conzemius, R. B. Evans, and M. J. Ritter, "Distribution of vertical forces in the pads of Greyhounds and Labrador retrievers during walking," American Journal of Veterinary Research, vol. 65, no. 11, pp. 1479-1501, 2004.

[30] R. M. Alexander and A. S. Jayes, "A dynamic similarity hypothesis for the gaits of quadrupedal mammals," Proceedings of the Zoological Society of London, vol. 201, no. 1, pp. 135-152, 1983.

[31] T. Wiestner, L. Galeandro, M. Hässig, P. M. Montavon, and K. Voss, "Effect of dog breed and body conformation on vertical ground reaction forces, impulses, and stance times," Veterinary and Comparative Orthopaedics and Traumatology, vol. 24, no. 2, pp. 106-112, 2011.

[32] W. C. Renberg, S. A. Johnston, K. Y. Ye et al., "Comparison of stance time and velocity as control variables in force plate analysis of dogs," American Journal of Veterinary Research, vol. 60, no. 7, pp. 814-819, 1998.

[33] M. Oosterlinck, T. Bosmans, F. Gasthuys et al., "Accuracy of pressure plate kinetic asymmetry indices and their correlation with visual gait assessment scores in lame and nonlame dogs," American Journal of Veterinary Research, vol. 72, no. 6, pp. 820-825, 2011.

[34] R. J. Corbee, H. Maas, A. Doornenbal, and H. A. W. Hazewinkel, "Forelimb and hindlimb ground reaction forces of walking cats: assessment and comparison with walking dogs," The Veterinary Journal, vol. 202, no. 1, pp. 116-127, 2014.

[35] E. Schnabl and B. Bockstahler, "Systematic review of ground reaction force measurements in cats," The Veterinary Journal, vol. 206, no. 1, pp. 83-90, 2015.

[36] J. A. Vilensky, "Locomotor behavior and control in human and non-human primates: comparisons with cats and dogs," Neuroscience \& Biobehavioral Reviews, vol. 11, no. 3, pp. 263-274, 1987.

[37] T. L. Quang, P. Maitre, A. Colin, and E. Viguier, "Spatial, temporal and kinetic evaluation of normal cats at walk, using a pressure walkway," Computer Methods in Biomechanics and Biomedical Engineering, vol. 11, no. 1, pp. 137-138, 2008.

[38] Y. Nakajima and F. Takahashi, "Increase of frictional force of rubber block by uniform contact pressure distribution and its application to tire," Rubber Chemistry and Technology, vol. 75, no. 4, pp. 589-604, 2002.

[39] A. C. B. C. F. Pinto, V. Marvulle, J. M. Matera, and A. N. A. Souza, "Evaluation of vertical forces in the pads of German Shepherd dogs," Veterinary and Comparative Orthopaedics and Traumatology, vol. 26, no. 1, pp. 6-11, 2013.

[40] M. Guillot, M. Moreau, M. A. d'Anjou, J. Martel-Pelletier, J.-P. Pelletier, and E. Troncy, "Evaluation of osteoarthritis in cats: novel information from a pilot study," Veterinary Surgery, vol. 41, no. 3, pp. 328-350, 2012.

[41] W. T. Kano, S. C. Rahal, L. R. Mesquita et al., "Gait analysis in a cat with scapular luxation and contralateral forelimb amputation," Canadian Veterinary Journal, vol. 54, no. 10, pp. 990-1000, 2013. 
[42] A. Pantall, R. J. Gregor, and B. I. Prilutsky, "Stance and swing phase detection during level and slope walking in the cat: effects of slope, injury, subject and kinematic detection method," Journal of Biomechanics, vol. 45, no. 8, pp. 15291533, 2012.

[43] B. D. X. Lascelles, K. Findley, M. Correa et al., "Kinetic evaluation of normal walking and jumping in cats, using a pressure-sensitive walkway," Veterinary Record, vol. 160, no. 15, pp. 512-516, 2007.

[44] S. C. Budsberg, M. C. Verstraete, and R. W. Soutas-Little, "Force plate analysis of the walking gait in healthy dogs," American Journal of Veterinary Research, vol. 48, no. 6, pp. 915-918, 1987.

[45] W. Tian, Q. Cong, and C. Menon, "Investigation on walking and pacing stability of German Shepherd dog for different locomotion speeds," Journal of Bionic Engineering, vol. 8, no. 1, pp. 18-24, 2011.

[46] M. Guillot, M. Moreau, M. Heit, J. Martel-Pelletier, J.-P. Pelletier, and E. Troncy, "Characterization of osteoarthritis in cats and meloxicam efficacy using objective chronic pain evaluation tools," The Veterinary Journal, vol. 196, no. 3, pp. 360-367, 2013.

[47] S. M. Stadig and A. K. Bergh, "Gait and jump analysis in healthy cats using a pressure mat system," Journal of Feline Medicine and Surgery, vol. 17, no. 6, pp. 523-529, 2014.

[48] F. S. Agostinho, N. S. M. L. Miqueleto, M. R. Verdugo, L. R. Inamassu, A. O. El-Warrak, and S. C. Rahal, "Kinematic analysis of labrador retrievers and rottweilers trotting on a treadmill," Veterinary and Comparative Orthopaedics and Traumatology, vol. 24, no. 3, pp. 185-191, 2011. 